\title{
Microexplosion and Puffing of an Emulsion Fuel Droplet
}

\author{
J. Xia*, J. Shinjo ${ }^{2}$ \\ ${ }^{1}$ Department of Mechanical, Aerospace and Civil Engineering \& Institute of Energy Futures, \\ Brunel University London, Uxbridge UB8 3PH, UK \\ 2 Department of Mechanical, Electrical and Electronic Engineering, \\ Shimane University, 1060 Nishikawatsu, Matsue 690-8504, Japan \\ *Corresponding author: jun.xia@brunel.ac.uk
}

\begin{abstract}
Microexplosion is rapid disintegration of an emulsion droplet caused by explosive boiling of embedded liquid subdroplets with a lower boiling point. Microexplosion and puffing (partial microexplosion) are potentially beneficial to achieving enhanced secondary atomisation. These eruptive secondary atomisation mechanisms will help to meet conflicting requirements for an atomising fuel spray with regard to penetration achieved by large droplets and evaporation/mixing achieved by small droplets.

Although with great interest, our understanding of microexplosion is still limited and therefore optimising and controlling microexplosion is not feasible yet. This paper reviews our recent research outcome on microexplosion and puffing of an emulsion fuel droplet from high-fidelity interface-capturing simulation study, which directly resolves the dynamics of boiling and evaporating liquid/gas interfaces, immiscible liquid/liquid interfaces with jump conditions for mass, momentum and heat transfer across a resolved interface.

We first unveiled microexplosion and puffing dynamics of an emulsion fuel droplet in a quiescent ambient. Since convective heating has important effects on temperature distribution inside a fuel droplet in realistic engine conditions, which determines the initial nucleation location and thus the microexplosion outcome, a model has been proposed to approximate the temperature distribution inside a droplet, for which momentum and heat transport was found to be only moderately correlated under typical engine conditions. With this model in place that allows for saving considerable computational cost on setting up initial conditions for microexplosion simulation, puffing effects on fuel/air mixing is then investigated, which can be quantified by the scalar dissipation rate (SDR) of the primary fuel decane, the SDR of the secondary fuel ethanol and the cross SDR. We then further extended our simulation studies towards reacting conditions and investigate puffing effects on a droplet wake flame.

The series of high-fidelity simulation studies is believed to have considerably improved our understanding of microexplosion dynamics and impact on local fuel/air mixing and combustion. Based on the improved knowledge, microexplosion induced secondary droplet breakup models can be developed and incorporated into hybrid highfidelity simulation of spray atomisation and combustion enhanced by microexplosion.
\end{abstract}

\section{Keywords}

Microexplosion; Puffing; Emulsion droplet; Mixing; Droplet combustion.

\section{Introduction}

Liquid-fuel spray atomisation and combustion is widely used in combustion engines. For engines, fuel efficiency is critical and emission regulations are more and more stringent. Using emulsion fuels, which can be water-in-oil or biofuel-in-fossil-fuel emulsions, can be an effective way to enhance fuel efficiency and reduce emissions. Emulsion fuel is an immiscible liquid mixture with sub-droplets dispersing in the primary liquid fuel. If physical properties such as the boiling point are distinctly different between the two immiscible liquids, emulsion fuels may show particular physical phenomenon, e.g. microexplosion, when injected into the combustion chamber. Microexplosion can be another important secondary breakup mechanism and play an important role in accelerating spray atomisation.

After injected into the combustion chamber, sprayed emulsion droplets will be heated by the high-temperature ambient. Take water-in-oil emulsions as an example. Since the boiling point of oil is typically higher than that of water, embedded water sub-droplets can be superheated, i.e. the water temperature will become higher than its boiling temperature. This state is physically metastable. With little disturbance, water starts to boil explosively. Within a short time period, explosive boiling can result in violent breakup of the parent droplet. Therefore microexplosion is rapid disintegration of an emulsion droplet caused by explosive boiling of embedded sub-droplets with a lower boiling point $[1,2,3]$. If the degree of breakup is limited, it is often termed as puffing.

Microexplosion and puffing have a great potential for enhancing secondary atomisation. These eruptive secondary atomisation mechanisms will help to meet conflicting requirements for an atomising fuel spray with regard to a deeper penetration achieved by large droplets and more efficient evaporation/mixing achieved by small droplets.

Although with a great potential, our understanding of secondary atomisation induced by microexplosion and puffing is still far from the stage at which they can be controlled and optimised. It is therefore vitally important to better understand the physics of microexplosion and puffing and their impact on fuel/air mixing and combustion, which is the aim of this paper.

To achieve this goal, we have performed a series of high-fidelity simulation study on a puffing emulsion droplet, which directly resolves boiling or evaporating liquid/gas interfaces and immiscible liquid/liquid interfaces. Interface 
capturing is based on a combined level-set and volume-of-fluid approach. The numerical method is based on the CIP (constrained interpolation profile) method [4,5], which is advantageous in many aspects for the simulation cases considered in the present study. Based on the knowledge gained, microexplosion induced secondary droplet breakup models can be developed and incorporated into hybrid high-fidelity simulation of spray atomisation and combustion enhanced by microexplosion.

\section{Methodology \\ Governing Equations}

The Navier-Stokes equations

$$
\dot{\mathbf{f}}+(\mathbf{u} \cdot \nabla) \mathbf{f}=\mathbf{S}
$$

are solved for the primitive variables $\mathbf{f}=\left[\rho, \mathbf{u}, T, Y_{i}\right]^{\mathrm{T}}$, where $\dot{\mathbf{f}}=\partial \mathbf{f} / \partial t$, and $\rho, \mathbf{u}, T$ and $Y_{i}$ denote density, velocity, temperature and the mass fraction of the $i$-th species, respectively. In addition to other general terms in NavierStokes excluding convection, $\mathbf{S}$ also includes the source terms due to surface tension, boiling and evaporation, and combustion in a reacting case. Level set functions are used to capture interfaces [6]. The level-set approach is combined with the MARS (Multi-interface Advection and Reconstruction Solver) [7] to improve mass conservation. Surface tension is modelled by the CSF (Continuum Surface Force) method [8]. Jump conditions [9, 10] are used to compute the heat, momentum and mass transfer across an interface. The details can be found in $[11,12,13,14]$.

\section{Numerical Procedures}

The governing equations are solved by a fractional step approach, which comprises two solving phases. In the advection phase, $\dot{\mathbf{f}}+(\mathbf{u} \cdot \nabla) \mathbf{f}=\mathbf{0}$ is solved using the CIP method. In the non-advection phases, $\dot{\mathbf{f}}=\mathbf{S}$ is solved using the CCUP (CIP-combined and unified procedure) method, which uses a pressure-based algorithm.

In the solver, pressure is first solved using a Poisson equation,

$$
\nabla \cdot\left(\frac{\nabla p^{*}}{\rho^{n}}\right)=\frac{p^{*}-p^{n}}{\rho^{n} c_{s}^{2} \triangle t^{2}}+\frac{\nabla \cdot \mathbf{u}^{\mathbf{n}}}{\triangle t}
$$

where the superscripts $n$ and $*$ indicate the the current time step and the time stage after the non-advective phase, respectively. $c_{s}$ is the adiabatic speed of sound. $\mathbf{u}^{*}, \rho^{*}, T^{*}$ and $Y_{i}^{*}$ can then obtained through $\dot{\mathbf{f}}=\mathbf{S}$ via the approximation $\mathbf{f}^{*}=\mathbf{f}^{n}+\triangle \mathbf{f}=\mathbf{f}^{n}+\mathbf{S} \triangle t$.

In the CIP method, the Lagrangian invariant $f(\mathbf{x}, t)=f(\mathbf{x}-\Delta \mathbf{x}, t-\triangle t)$ is employed. The "constraint" is set up by the first derivative of $f$, which follow an advection equation derived from $\dot{f}+(\mathbf{u} \cdot \nabla) f=0$. It is now well known that if a constraint is independent of the original governing equation, it will not help to retrieve the profile inside a grid cell [5]. Knowing $f$ and its derivative $f^{\prime}$ only at two grid points comprising a grid cell, a cubic polynomial is used to approximate the subgrid or subcell solution of $f$ and $f^{\prime}$ by enforcing their continuities at all the grid points $[4,5]$.

The numerical scheme therefore features compact support and subgrid resolution. It can preserves the sharpness of an interface where large gradients of density exist and does not need an adaptive grid. It is free from the CFL condition that is restricted by a high sound speed. With the two-stage fractional step approach, it can solve incompressible flow in the framework of compressible fluid, e.g. the interaction between a compressible gas and incompressible liquid or solid.

\section{Results and discussion}

Puffing Dynamics [11]

Direct Simulation of Puffing Figures 1 and 2 present the initial puffing and subsequent after-puffing dynamics of two representative two-dimensional cases, in which the ratio of the radius of the low-boiling-point sub-droplet to that of the high-boiling-point parent droplet is $R_{\mathrm{sub}} / R_{\mathrm{p}}=0.25$ in Fig. 1 and 0.35 in Fig. 2, respectively. The other initial parameters are identical for the two cases. Due to the availability of published data on water-in-oil emulsions, in this subsection we use hexadecane and water properties for the parent and sub droplets, respectively. The ambient air pressure is $3 \mathrm{MPa}$, and the initial temperature is $553 \mathrm{~K}$. Since the water boiling temperature is $503 \mathrm{~K}$, the superheat degree is $50 \mathrm{~K}$. The initial velocity is 0 . The initial diameter of the parent droplet is $D_{\mathrm{p}}=30 \mu \mathrm{m}$. The computational domain size is $4.2 D_{\mathrm{p}} \times 4.2 D_{\mathrm{p}}$ discretised with $381 \times 381$ grid points. The minimum grid spacing is $0.26 \mu m$. Open boundaries are used.

Initially, the vapour bubble near the water/oil interface grows due to boiling. Since the liquid oil "wall" between the boiled vapour and the parent droplet surface is thin, the rapidly growing bubble will push the wall strongly, leading to rupture of the parent droplet, puffing of boiled vapour and ejection of liquid oil fragments [Figs. 1(a) and 2(a)]. After puffing, the boiling surface continues to eject vapour, which exerts a thrust force on the sub-droplet itself.

In the confined region where the newly formed water/vapour liquid/gas interface interacts with the water/oil liquid/liquid interface as indicated by blue arrows in Figs. 1(b) and 2(b), defined herein as an "edge", the water/vapour interface becomes unstable due to the Landau-Darrieus instability and the troughs therefore deepen. The edge regression proceeds, so that boiled vapour wrap up the sub-droplet, which finally detaches itself from the parent droplet. After the detachment, the sub-droplet stops affecting the parent droplet and the puffing-induced breakup process mostly ends.

The major difference between the two cases is the time period for the detachment of the whole sub-droplet from the parent droplet after puffing occurs is longer in Fig. 2 than in Fig. 1. It was found that for a big sub-droplet, its 


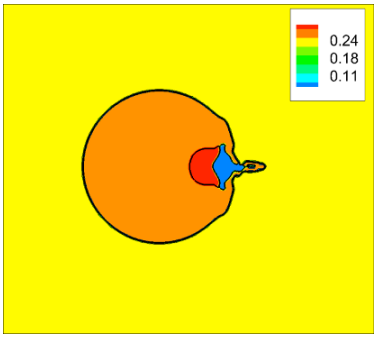

(a) $t=0.4 \mu \mathrm{s}$

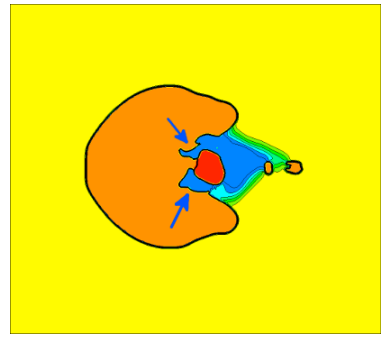

(b) $t=1.4 \mu \mathrm{s}$

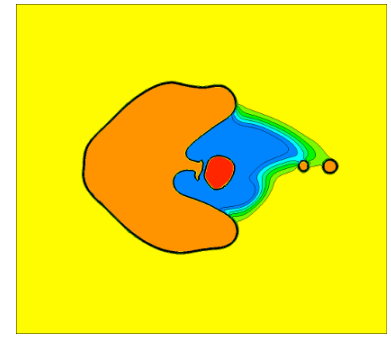

(c) $t=2.5 \mu \mathrm{s}$

Figure 1. Puffing and after-puffing due to boiling of a small sub-droplet $\left(R_{\mathrm{sub}} / R_{\mathrm{p}}=0.25\right)$ [11], where $R_{\mathrm{sub}}$ and $R_{\mathrm{p}}$ are the radius of the sub- and parent droplets, respectively. Orange: parent droplet with a higher boiling point. Red: sub-droplet with a lower boiling point. Gas-phase colour contours: mass fraction of oxygen $Y_{\mathrm{O}_{2}}$. Accordingly, blue: boiled vapour \& yellow: air.

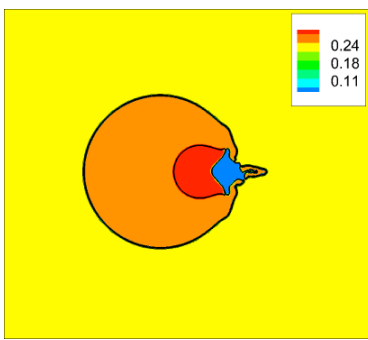

(a) $t=0.4 \mu \mathrm{s}$

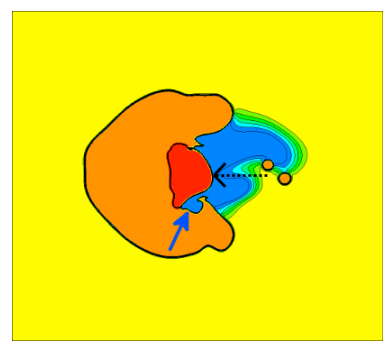

(b) $t=2.2 \mu \mathrm{s}$

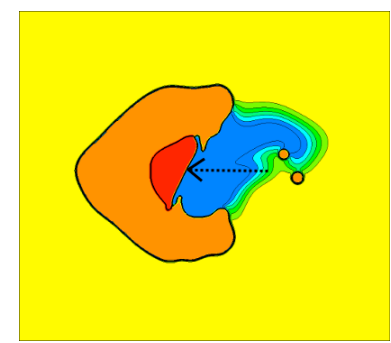

(c) $t=3.2 \mu \mathrm{s}$

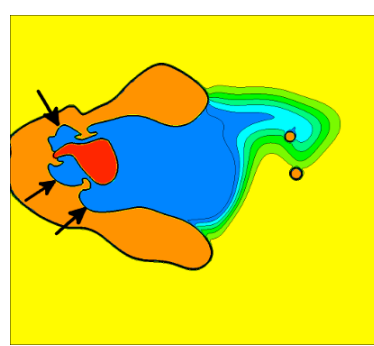

(d) $t=5.5 \mu \mathrm{s}$

Figure 2. Puffing and after-puffing with a big sub-droplet $\left(R_{\mathrm{sub}} / R_{\mathrm{p}}=0.35\right)$ [11]. See Fig. 1 for colour and variable definitions.

boiling surface oscillates strongly and its shape varies accordingly. As shown in Fig. 2(b) and 2(c) by a dashed arrow, the shape of the boiling surface is flattened due to the oscillation and some detached part of the sub-droplet remerges with the parent droplet. The interface edge regression is thus suspended and then restarts. For a smaller sub-droplet in Fig. 1, no remerging was seen and therefore the time period of the sub-droplet detachment is shorter.

Edge Regression A boiling surface exhibits the Landau-Darrieus (LD) instability due to evaporating mass flux at the interface [15] (Fig. 3). Due to the evaporating mass flux, the streamlines are bent at the liquid troughs and crests, working to enhance the interface shape deformation. Even if no vorticity exists in the liquid phase, vorticity will be generated in the gas phase, as illustrated by the dashed arrows in Fig. 3. Vorticity generation in the gas phase has been found to play an important role in the instability development and the detachment of a sub-droplet from the parent droplet after puffing occurs.

Figure 4 shows the vorticity component normal to the paper before and after puffing, with positive and negative vorticities indicating counterclockwise and clockwise rotations, respectively. At $t=0.2 \mu \mathrm{s}$ when puffing will soon occur, the vapour bubble is pushing the thin liquid "wall" towards the right direction. The observed strong symmetric vorticity is due to this outward jet-like flow but not the LD instability. The boiling surface of the sub-droplet is also pulled towards the same direction by the jet flow. As a consequence, the concave shape of the boiling surface becomes convex after puffing occurs, as indicated by the red arrow in Fig. 4(a) and 4(b). This induces oscillation of the sub-droplet.

Vorticity structures near the edges in a trough shape are indicated by small solid black arrows. An "edge" is formed at the intersection among the oil/water liquid/liquid interface, the non-boiling, inert oil/air liquid/gas interface and the boiling water/vapour liquid/gas interface. At $t=0.8 \mu \mathrm{s}$ and $t=1.6 \mu \mathrm{s}$, positive and negative vorticity pairs are observed in the concave regions near an edge. The vorticity generation is due to the local evaporating mass flux from the boiling surface. Note in the immediate vicinity the oil/vapour interface is non-boiling and inert, and therefore does not contribute to vorticity generation. The vorticity pairs work to deepen the trough, as shown in Figs. 4 and 5. Therefore, the initiation of edge regression is due to the LD instability.

After edge regression is initiated, the boiling surface of the sub-droplet gradually extends and regresses towards inside. During this regression process, bubble-like structures grow in the edge regions and a wavy pattern forms on the detached inert interface between the boiled vapour and the liquid species (oil in this case) of the parent droplet, as indicated by bold black arrows in Fig. 4(b) and 4(c), also illustrated in Fig. 5 and can be seen in Fig. 2(d). This is induced by the Rayleigh-Taylor (RT) instability, the mechanism of which is due to density difference and surface acceleration. In the neck regions created by the RT instability, the local ejecting vapour is accelerated by the "nozzle" effect and the vorticity magnitude is large. The vapour flow also pushes the developed RT "plumes" 


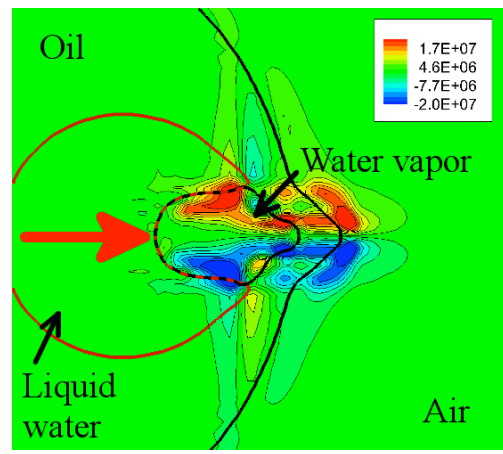

(a) $t=0.2 \mu \mathrm{s}$

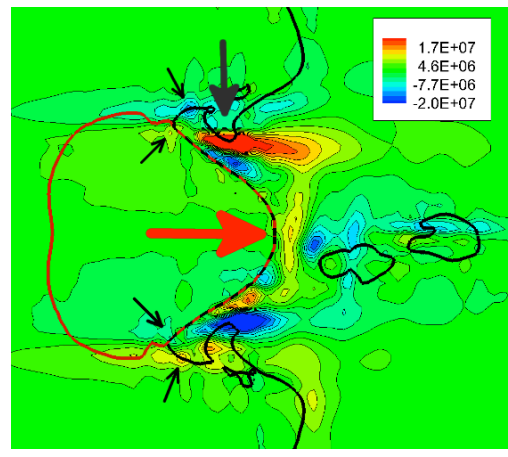

(b) $t=0.8 \mu \mathrm{s}$

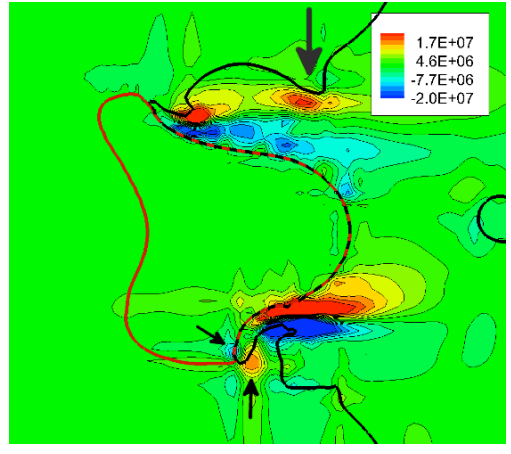

(c) $t=1.6 \mu \mathrm{s}$

Figure 4. Vorticity generation due to Landau-Darrieus instability: an important mechanism for edge regression [11]. Colour contours: vorticity $\left(s^{-1}\right)$.

outward. Following these physical processes, the edge regression proceeds and the sub-droplet will eventually detach itself from the parent droplet.

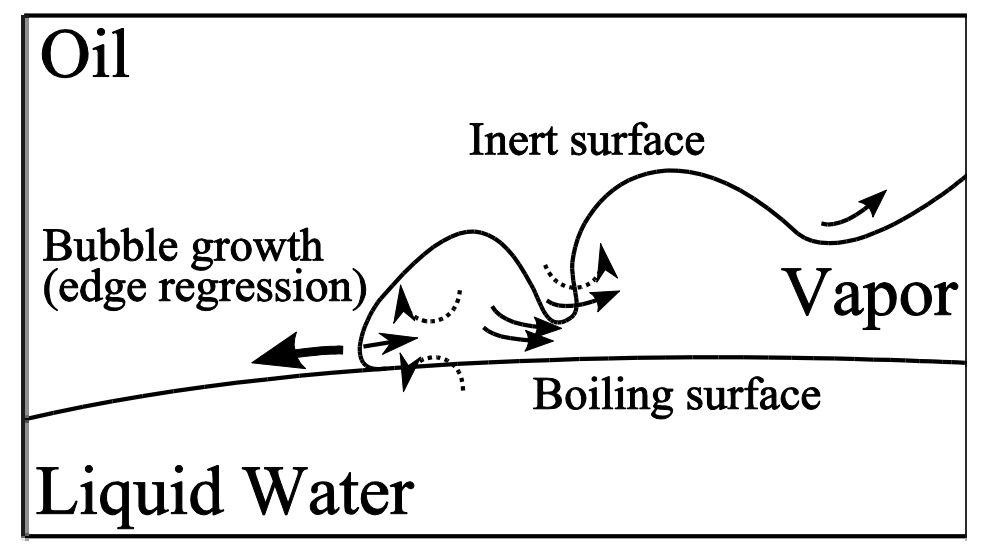

Figure 5. Schematic of edge regression mechanism [11] according to Fig. 4. The Landau-Darrieus instability develops due to the boiling surface. The Rayleigh-Taylor instability develops on the inert surface. Their interactions lead to edge regression.

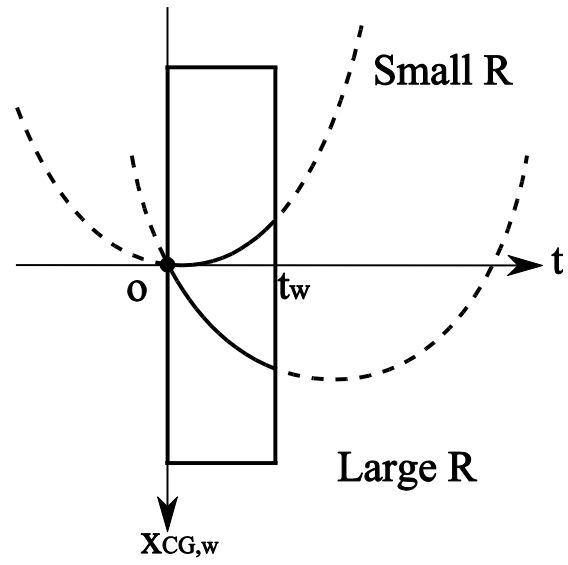

Figure 6. Centre of gravity $x_{\mathrm{CG}, \mathrm{w}}$ of a small and large sub-droplet, indicating whether a one-stage detachment or a two-stage penetration/detachment occurs for the sub-droplet following puffing [11]. At $t_{\mathrm{w}}$, edge regression completes and the entire sub-droplet detaches from the parent droplet.

One-Stage or Two-Stage Detachment An approximate quantitative analysis has been attempted in [11] to investigate how the distance $d_{\mathrm{ow}}$ between the centre of gravity of the parent droplet $\left(x_{\mathrm{CG}, \mathrm{o}}\right)$ and that of the sub-droplet $\left(x_{\mathrm{CG}, \mathrm{w}}\right)$ varies after puffing occurs. Neglecting the movement of the parent droplet, $d_{\mathrm{ow}}=x_{\mathrm{CG}, \mathrm{o}}-x_{\mathrm{CG}, \mathrm{w}}=$ const. $-x_{\mathrm{CG}, \mathrm{w}}$, so $d_{\mathrm{ow}}$ is determined by $x_{\mathrm{CG}, \mathrm{w}}$. The analysis included effects of the inertia of the bubble burst (or the pulling effect) and the thrust force generated by boiling, but excluded sub-droplet shape oscillation. It was found that $x_{\mathrm{CG}, \mathrm{w}}$ can be approximately predicted by $x_{\mathrm{CG}, \mathrm{w}}=-A t^{2}+B(t-C)$, where $A, B$ and $C$ are constants, and $t$ is time. If the sub-droplet size $R_{\text {sub }}$ is small, the first term $-A t^{2}$ due to the pulling effect will be dominant, and thus the sub-droplet tends to move away from the parent droplet, so the detachment process proceeds by edge regression. On the other hand if $R_{\text {sub }}$ is big, the second term $B(t-C)$ due to the thrust will play the dominant role and the subdroplet tends to penetrate towards the parent droplet, leading to partial remerging of the sub- and parent droplets, temporary suspension of edge regression and thus a longer time period for the sub-droplet detachment from the parent droplet. Figure 6 summarises the above findings. It shows that $x_{\mathrm{CG}, \mathrm{w}}$ can have opposite signs for a small and large sub-droplet.

Therefore the direction of the sub-droplet motion determines whether a one-stage detachment or a two-stage penetration/detachment occurs for the sub-droplet following puffing. In either case, the sub-droplet starts shape oscillation after puffing. Only if penetration occurs when the thrust effect is dominant, the oscillating boiling surface will be flattened [see Fig. 2(b) and 2(c)] and partial remerging of the sub- and parent droplets will cancel out part of the already generated boiling surface, leading to a decrease and fluctuation of the boiling surface area $S_{\text {boil }}$ (not shown). Since the edge instability mechanisms exists as long as boiling continues, the surface regression restarts after the remerging until the sub-droplet detaches completely from the parent droplet and the puffinginduced breakup ends. 


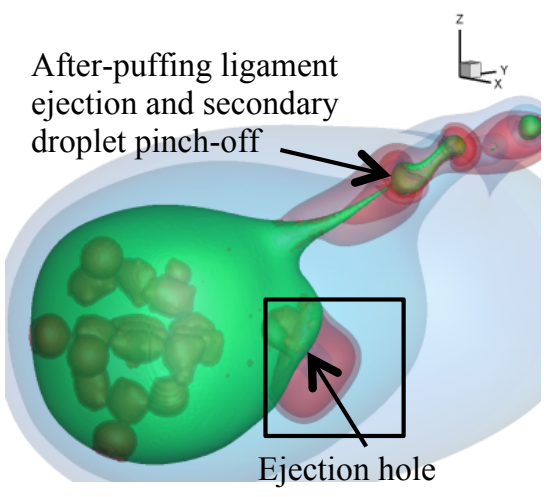

(a) $N_{\text {nuclei }}=2$

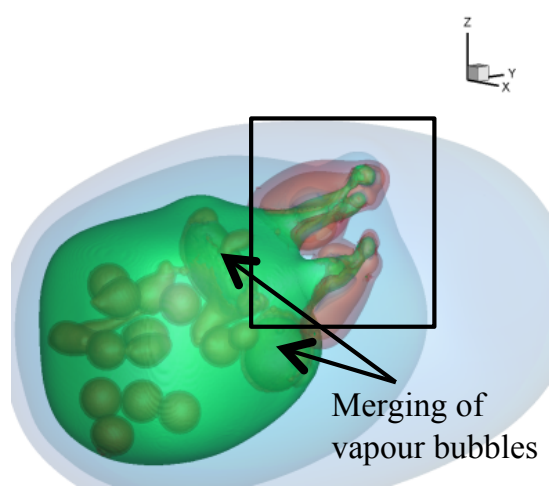

(b) $N_{\text {nuclei }}=4$

Figure 7. Puffing of an ethanol-in-decane emulsion droplet under convective heating [13]. $N_{\text {nuclei }}$ is the number of initial nuclei. Vapour of the primary fuel decane is produced at the surface of the parent droplet through evaporation modelled by the Clapeyron-Clausius law. Vapour of the secondary fuel ethanol is produced by sub-droplet boiling and participates in fuel/air mixing after puffing occurs. The two blue iso-surfaces indicate $Y_{\text {decane }}=Y_{1}=0.1$ and 0.2 , and the two red iso-surfaces $Y_{\text {ethanol }}=Y_{2}=0.05$ and 0.1 . The rectangles shows the regions used for analysis of (a) weak puffing and (b) strong puffing accompanying ejections of liquid-fuel ligaments.

(a)

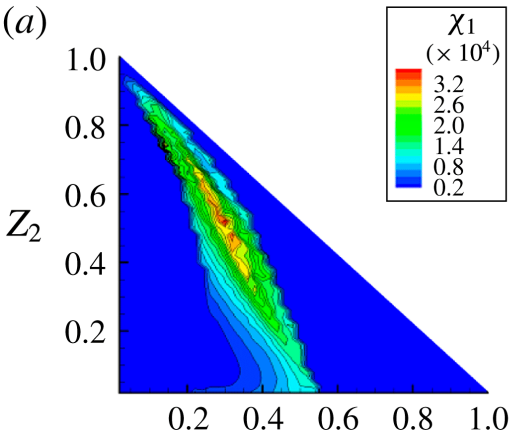

(b)

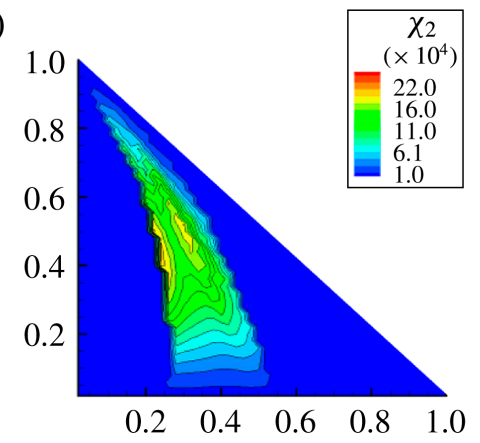

(c)

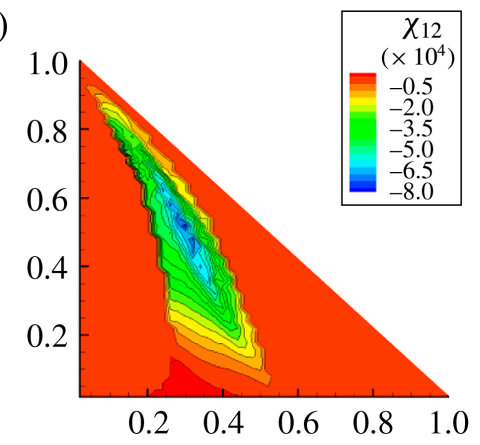

Figure 8. Doubly conditioned mean scalar dissipation rate $\chi$ in weak puffing with no ejection of liquid-fuel ligaments [13]: (a) $\left\langle\chi_{1} \mid Z_{1}, Z_{2}\right\rangle$; (b) $\left\langle\chi_{2} \mid Z_{1}, Z_{2}\right\rangle$; (c) $\left\langle\chi_{12} \mid Z_{1}, Z_{2}\right\rangle$. Z: mixture fraction. Subscript 1: primary fuel decane. Subscript 2: secondary fuel ethanol. Analysis region: rectangle indicated in Fig. $7(\mathrm{a})$. It should be stressed that the contour legends are different.

(a)

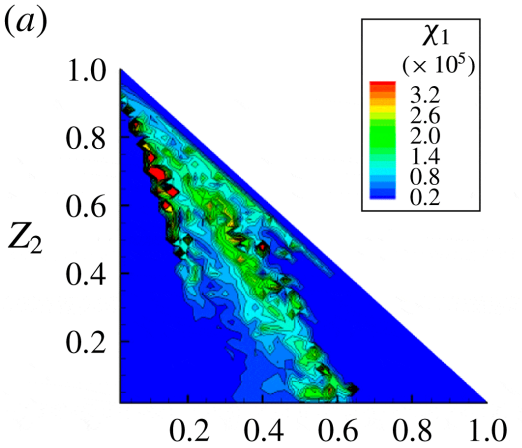

(b)

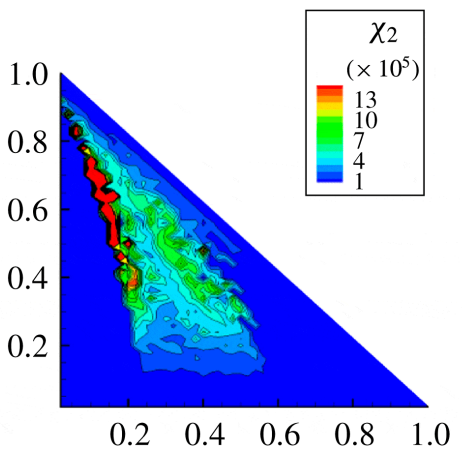

(c)

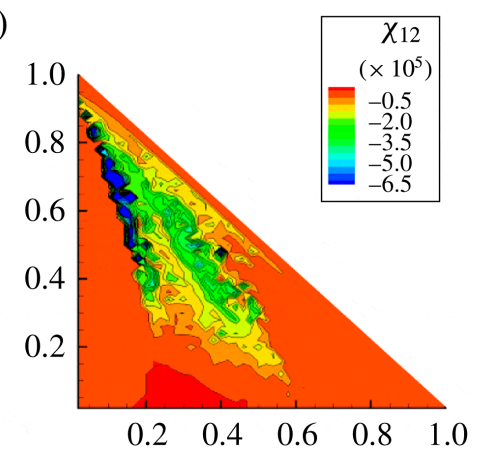

Figure 9. Doubly conditioned mean scalar dissipation rate $\chi$ in strong puffing with ejections of liquid-fuel ligaments [13]: (a) $\left\langle\chi_{1} \mid Z_{1}, Z_{2}\right\rangle$; (b) $\left\langle\chi_{2} \mid Z_{1}, Z_{2}\right\rangle$; (c) $\left\langle\chi_{12} \mid Z_{1}, Z_{2}\right\rangle$. See Fig. 8 for variable definitions. Analysis region: rectangle indicated in Fig. 7 (b).

\section{Puffing-Enhanced Fuel/Air Mixing [13]}

Figure 7 presents puffing of an ethanol-in-decane emulsion droplet under convective heating. The only difference between the two cases is the number of initial nuclei $N_{\text {nuclei }}$. With more initial nuclei, ethanol vapour puffing is expected to be more intense. To better understand the effects of puffing on fuel/air mixing, we classify puffing into weak and strong puffing, in which cases liquid-fuel ligaments do not and do accompany the puffing, respectively. Therefore the region indicated by the rectangle in Fig. 7(a) and Fig. 7(b) has been used for analysis of weak and strong puffing.

The diameters of the parent decane droplet and the ethanol sub-droplet are $D_{\mathrm{p}}=30 \mu \mathrm{m}$ and $D_{\text {sub }}=4.6 \mu \mathrm{m}$, respectively. The number of ethanol sub-droplets in a parent droplet is 19 , and therefore the volume fraction of ethanol is $7.4 \%$. The free-stream air velocity is $U_{\mathrm{g}}=10 \mathrm{~m} / \mathrm{s}$, the air temperature $T_{\mathrm{g}}=900 \mathrm{~K}$, and the ambient air pressure $p_{\mathrm{g}}=1 \mathrm{MPa}$. The droplet Reynolds number is $R e_{\mathrm{d}}=30$. At $1 \mathrm{MPa}$, the boiling temperature of 

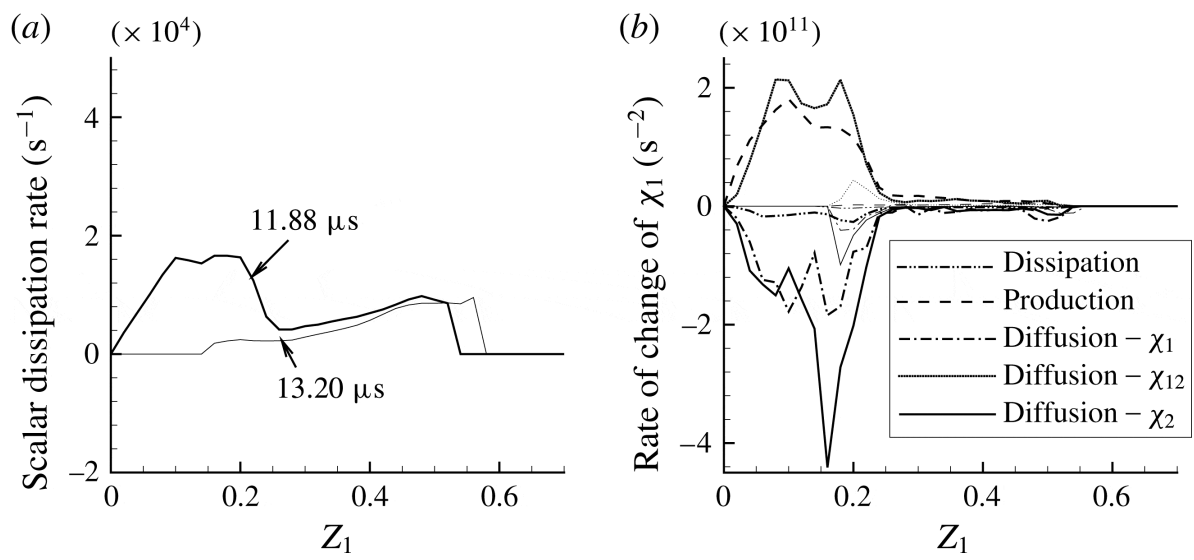

Figure 10. Puffing-enhanced micro-mixing [13]. (a) $\left\langle\chi_{1} \mid Z_{1}\right\rangle$; (b) budget analysis on $\left\langle\chi_{1} \mid Z_{1}\right\rangle$ using Eq. 3.

decane and ethanol is $565 \mathrm{~K}$ and $425 \mathrm{~K}$, respectively. The superheat limit of ethanol is $477 \mathrm{~K}$. The grid spacing is $\Delta x=\Delta y=\Delta z=0.19 \mu \mathrm{m}$. The total number of grid points is 243 million.

The droplet heating process prior to puffing is much slower than puffing. Under convective heating, internal circulation is developed inside an (emulsion) droplet. It was found that under typical flow conditions inside a combustion chamber $\left(R e_{\mathrm{d}} \sim 30\right)$, the liquid Péclet number is in a transitional regime $\left(100<P e_{\mathrm{L}}<500\right)$. Therefore models developed for inner-droplet velocity distribution $[16,17]$ cannot be borrowed to approximate temperature distribution. A new model was therefore developed [12] to approximate the inner-droplet temperature distribution and used here to save computational cost on droplet heating.

A mixture fraction, $Z_{i}=\left(Y_{i}-Y_{i, \mathrm{FAR}}\right) /\left(Y_{i, \mathrm{CTR}}-Y_{i, \mathrm{FAR}}\right)$ [18], has been used to quantify the fuel-vapour/air mixing without combustion, where $i=1$ and 2 indicates the primary fuel decane and the secondary fuel ethanol, respectively. Setting $Y_{i, \mathrm{FAR}}=0$ in the far field and $Y_{i, \mathrm{CTR}}=1$ at the droplet centre, $Z_{i}$ is equivalent to $Y_{i}$. The scalar dissipation rate, $\chi$, is used to quantify the micro-mixing and defined as $\chi_{i j}=2 D_{\text {diffu }} \nabla Z_{i} \cdot \nabla Z_{j}$, where $D_{\text {diffu }}$ is the diffusion coefficient. Therefore $\chi_{1}, \chi_{2}$ and $\chi_{12}$ quantify the primary-fuel diffusion, secondary-fuel diffusion and cross-diffusion of the two fuels in the gas phase.

Figures 8 and 9 present the SDRs doubly conditioned on $Z_{1}$ and $Z_{2}$, i.e. $\left\langle\chi_{1} \mid Z_{1}, Z_{2}\right\rangle$ and $\left\langle\chi_{2} \mid Z_{1}, Z_{2}\right\rangle$, and the cross-SDR $\left\langle\chi_{12} \mid Z_{1}, Z_{2}\right\rangle$ at the initial stage of puffing for weak [Fig. 7(a)] and strong [Fig. 7(b)] puffing, respectively. It should be noted that the upper limit of the mixture fractions in the figures is bounded by $Z_{1}+Z_{2}=1$ by definition. Immediately after the ethanol vapour ejection starts, $\chi_{1}$ and $\chi_{2}$ increase. It is also interesting to see that $\chi_{2}$ is one order of magnitude bigger than $\chi_{1}$, indicating that strong stratification of ethanol vapour exists in the gas phase once puffing occurs. Cross-mixing of the decane and ethanol fuel vapour is also evident, as $\chi_{12}$ is negative. The SDRs in the region of large values of $Z_{2}$ are due to ethanol vapour ejection, and those in the region of large valued of $Z_{1}$ are due to decane evaporation at the parent droplet surface. It should be noted that the upper limit of $Y_{1}$ and $Y_{2}$ is the local saturated vapour pressure dictated by the the Clapeyron-Clausius model and 1 due to boiling, respectively. For strong puffing shown in Fig. 9, the magnitudes of SDRs are larger, and the SDR values are more strongly disturbed than in Fig. 8 due to multiple ejections and ejected liquid fragments.

Since the primary fuel is decane, puffing effects on $\chi_{1}$ and $Z_{1}$ are of primary interest. Assuming that the mixing is dominant in the compressive strain direction [19], the transport equation for $\chi_{1}$ in the mixture fraction space can be written as

$$
\frac{\partial \chi_{1}}{\partial t}=\underbrace{2 a \chi_{1}}_{\text {Production }}-\underbrace{\frac{1}{4}\left(\frac{\partial \chi_{1}}{\partial Z_{1}}\right)^{2}}_{\text {Dissipation }}+\underbrace{\frac{\chi_{1}}{2} \frac{\partial^{2} \chi_{1}}{\partial Z_{1}^{2}}}_{\begin{array}{c}
\text { Primary-fuel } \\
\text { diffusion }
\end{array}}+\underbrace{\chi_{12} \frac{\partial^{2} \chi_{1}}{\partial Z_{1} \partial Z_{2}}}_{\text {Cross-diffusion }}+\underbrace{\frac{\chi_{2}}{2} \frac{\partial^{2} \chi_{1}}{\partial Z_{2}^{2}}}_{\begin{array}{c}
\text { Seondary-fuel } \\
\text { diffusion }
\end{array}}+\underbrace{S_{\chi_{1}}}_{\begin{array}{c}
\text { Evaporation } \\
\text { source }
\end{array}},
$$

where $a$ is the dominant compressive strain rate. The compressive strain rate makes the local gradients steeper, which contributes positively to the rate of change of the SDR. The dissipation term is always negative. The effect of the evaporation source term $S_{\chi_{1}}$ is confined in the vicinity of an evaporating/boiling surface. It is clear from Eq. 3 that the additional terms due to the dissipation rate $\chi_{2}$ and cross-SDR $\chi_{12}$ quantify the direct puffing effects on the mixing and micro-mixing of the vapour of the primary fuel decane with air.

Figure 10(a) presents the conditional mean SDR $\left\langle\chi_{1} \mid Z_{1}\right\rangle$ for the weak puffing case. At $t=11.88 \mu s$ [Fig. 7(a)], enhanced mixing due to the ejected ethanol vapour is evident in $Z_{1}=0.1-0.25$. The other peak in the regions of large values of $Z_{1}$ is due to decane evaporation from the parent droplet. At a later time $t=13.20 \mu \mathrm{s}$, the puffing jet has already stopped and $\chi_{1}$ becomes much smaller in $Z_{1}=0.1-0.25$, which indicates that puffing-induced mixing has weakened. Therefore, puffing enhances mixing in a short period of the ethanol vapour ejection.

The budget analysis in Fig. 10(b) shows the contributions of each conditionally averaged right-hand-side term in Eq. 3 to $\partial\left\langle\chi_{1} \mid Z_{1}\right\rangle / \partial t$. The evaporation source term is not included in the analysis. The production term $2 a \chi_{1}$ is approximated by $2 S \chi_{1}$, where $S$ is the norm of the strain rate tensor $S_{i j}=0.5\left(\partial u_{i} / \partial x_{j}+\partial u_{j} / \partial x_{i}\right)$.

It can be seen that the dissipation term is a sink by definition and its magnitude is small. A leading source is the production term due to the strong strain rate generated by the ejecting ethanol vapour jet. It is interesting to 
note that the cross-diffusion due to $\chi_{12}$ is another leading source that is comparable to the production term. This indicates that puffing-induced cross-diffusion of the primary and secondary fuels works to increase the magnitude of $\chi_{1}$ in the full spectrum of $Z_{1}$. The other two diffusion terms are negative, which represents a generic diffusion process. At $t=11.88 \mu \mathrm{s}$, puffing is intense with mixing in progress. The net contribution of all the right-hand-side terms is negative, which decreases $\chi_{1}$. At a later time $t=13.20 \mu \mathrm{s}$, the magnitudes of these terms are smaller, indicating that the puffing jet has stopped and the fuel/air mixing has progressed further.

The same budget analysis was also performed for strong puffing [Fig. 7(b)] (not shown). An important difference from Fig. $10(\mathrm{~b})$ is that the cross-scalar diffusion due to $\chi_{12}$ now takes the dominant role in enhancing $\left\langle\chi_{1} \mid Z_{1}\right\rangle$, whereas in the weak puffing case the production and cross-scalar diffusion are comparable.

Through the above detailed analysis, it is clear that ejection of boiled ethanol vapour initially generates a strong stratification in the reactive gaseous mixture of decane vapour and air, followed by mixing that homogenises the stratification. Intermittent ethanol vapour puffing therefore modifies local mixing characteristics around the emulsion droplet both in time and in space.

\section{Droplet Combustion under Puffing [14]}

Figure 11 shows a snapshot of puffing and puffing-induced interaction between ejected ethanol vapour and a decane-droplet wake flame at $t=9.2 \mu \mathrm{s}$. Combustion of decane and ethanol is modelled by a global one-step chemistry mechanism. Six species, i.e. decane $\left(\mathrm{C}_{10} \mathrm{H}_{22}\right)$, ethanol $\left(\mathrm{C}_{2} \mathrm{H}_{5} \mathrm{OH}\right), \mathrm{O}_{2}, \mathrm{~N}_{2}, \mathrm{CO}_{2}$ and $\mathrm{H}_{2} \mathrm{O}$, are considered. The other simulation parameters are the same as in the previous subsection. Ethanol vapour ejection starts at $t=5.5 \mu \mathrm{s}$ following bubble nucleation and growth. The ejected ethanol vapour reaches the flame surface and interactions between the flame and ethanol vapour start shortly before $t=7.8 \mu \mathrm{s}$. At $t=9.2 \mu \mathrm{s}$, the flame shape is locally stretched and distorted due to the flame/vapour interaction underway.

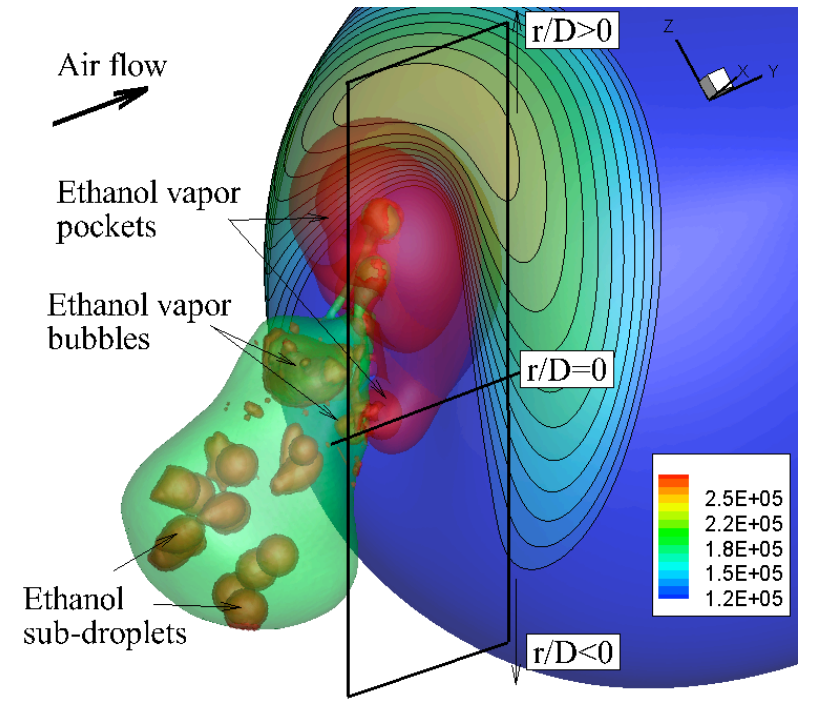

Figure 11. Puffing and puffing-induced interaction between ejected ethanol vapour and the decane-droplet wake flame at $t=9.2 \mu s$ [14]. Red iso-surfaces: $Y_{2}=Y_{\text {ethanol }}=0.05$ and 0.1 . Green iso-surface: parent-droplet surface. Dark green iso-surfaces: sub-droplet surfaces. Blue iso-surface:

$T_{\mathrm{g}}=1,800 \mathrm{~K}$, illustrating the droplet wake flame. Colour contours: ethanol consumption rate $\left(\mathrm{mol} / \mathrm{m}^{3} / \mathrm{s}\right)$.

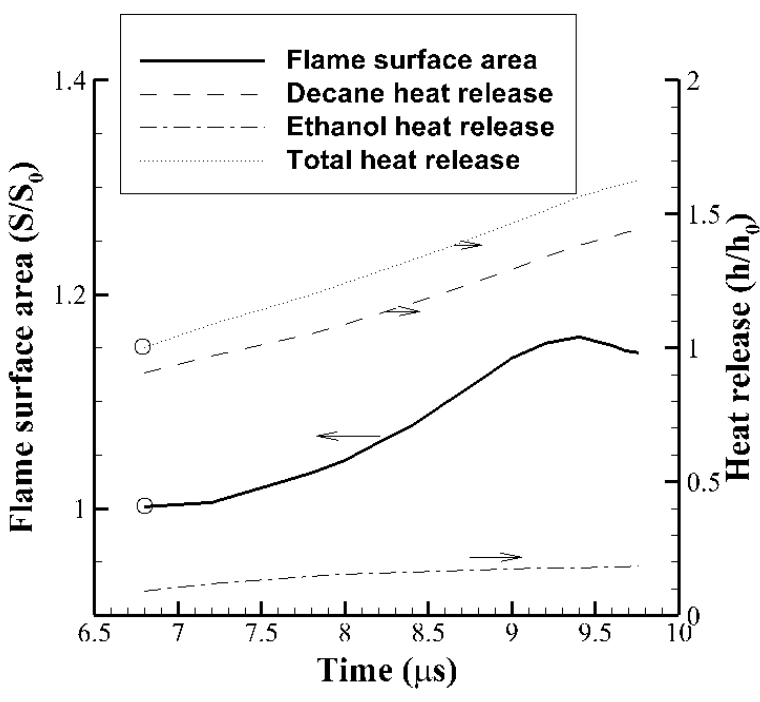

Figure 12. Temporal traces of flame surface area $S$, heat release rate of decane $h_{1}$ and ethanol $h_{2}$, and total heat release rate $h$ [14]. $S_{0}$ and $h_{0}$, indicated by the circles, are used for normalisation.

The global characteristics of the interaction between the puffing and the droplet flame is presented in Fig. 12, which shows the temporal traces of the normalised flame surface area $S / S_{0}$, the normalised heat release rates of the primary fuel decane and the secondary fuel ethanol, and their sum - the normalised total heat release rate. It is clear that the puffing transiently increases the flame surface area and also the total heat release rate. As diffusion progresses, the transient effect becomes weaker. The flame surface area reaches a maximum value at $t \approx 9.4 \mu s$ and decreases thereafter until the next ethanol vapour pocket arrives. The heat release rate reaches a maximum value at a later time than $t=9.5 \mu \mathrm{s}$ due to puffing-enhanced-mixing effects on combustion.

A mixture fraction is defined for fuel-droplet evaporation with combustion [18] as $Z_{i}=\left[Y_{i}+\left(Y_{\mathrm{O}_{2}}, \mathrm{FAR}-Y_{\mathrm{O}_{2}}\right) / s_{i}\right]$ $/\left(1+Y_{\mathrm{O}_{2}}, \mathrm{FAR} / s_{i}\right)$ for a one-step global reaction $\mathrm{F}_{i}+s_{i} \mathrm{O} \rightarrow\left(1+s_{i}\right) \mathrm{P}_{i}$, where $i=1$ and $i=2$ denote the primary fuel decane and the secondary fuel ethanol, respectively. $\mathrm{F}_{i}, \mathrm{O}$ and $\mathrm{P}_{i}$ stand for fuel, oxidiser and product, respectively. $s_{i}$ is the ratio of the mass of oxygen consumed per mass of fuel in a stoichiometric reaction. The budget analysis on the conditional mean scalar dissipation rate $\left\langle\chi_{1} \mid Z_{1}\right\rangle$ has been performed for the droplet wake flame under puffing using Eq. 3, and a qualitatively similar trend has been identified to the strong puffing case (without combustion) as shown in Fig. 7(b). 


\section{Conclusions}

This paper reviews our recent work on high-fidelity simulation study on microexplosion and puffing of an emulsion fuel droplet, including puffing dynamics and impact of puffing on local fuel/air mixing and emulsion-droplet combustion. A combined level-set and volume-of-fluid approach has been used for interface capturing, with jump conditions describing heat, momentum and mass transfer across an interface. The CIP method [4,5] was found to be efficient and effective to solve incompressible flow in the framework of compressible fluid, in our case the interaction between a compressible gas and incompressible liquid. Therefore boiling and evaporating droplet surfaces, liquid/liquid interfaces are directly resolved without the use of an adaptive grid.

Microexplosion and puffing dynamics are examined by direct simulation on a water-in-oil (approximated by hexadecane) emulsion droplet in a quiescent ambient. The growth of a single bubble, initiated at the oil/water interface, leads to puffing. The bubble burst and the sub-droplet dynamics after puffing jointly determine the oil-droplet breakup outcome.

After puffing, whether the detachment of a sub-droplet from the parent droplet is in one stage or two stages is determined by 3 competing mechanisms: (1) shape oscillation of the sub-droplet, (2) thrust generated by boiling, and (3) pulling effects due to the inertia of the bubble burst. If the sub-droplet is small, the pulling effect is dominant. The sub-droplet quickly detaches from the parent droplet due to edge regression, and the extent of the droplet breakup is therefore limited. If the sub-droplet is large, it penetrates into the parent droplet by the thrust. The mutual interaction between the sub- and parent droplets thus takes a longer time and the degree of breakup is larger.

In both one- and two-stage detachment, edge regression is a key mechanism for the sub-droplet detachment from the parent droplet. Through edge regression, the boiling surface of the sub-droplet gradually extends until boiling occurs at the whole oil/water interface, when the sub-droplet detaches itself from the parent droplet. The dominant mechanism is the Landau-Darrieus instability, which generates closely attached vorticity pairs with opposite signs due to the evaporating mass flux at the boiling surface. These vorticities then works to enlarge the vapour bubble area in the vicinity of the edge, which can then extend further towards the oil/water interface.

Puffing effects on fuel/air mixing are quantitatively analysed for weak puffing and strong puffing accompanying ejections of liquid-fuel ligaments in a convective heating flow. The transport equation for the scalar dissipation rate of the primary fuel decane is used. The two additional terms in the equation due to the dissipation rate $\chi_{2}$ of the secondary fuel ethanol and cross diffusion $\chi_{12}$ of the two fuels quantify the direct puffing effects on the mixing and micro-mixing of the vapour of the primary fuel decane with air. The two leading sources are found to be (1) the production terms due to the strong strain rate generated by ejecting ethanol vapour and (2) the cross-diffusion, which is much stronger than the production in strong puffing than in weak puffing. The detailed analysis demonstrates that ejection of boiled ethanol vapour initially generates a strong stratification in the reactive gaseous mixture of decane vapour and air, followed by mixing that homogenises the stratification. Intermittent ethanol vapour puffing therefore modifies local mixing characteristics around the emulsion droplet both in time and in space.

Using a global one-step chemistry mechanism, puffing effects on a droplet wake flame are investigated. The flame surface area and heat release rate are increased due to impact of ejected ethanol fuel vapour on the droplet flame. Budget analysis is also performed on the transport equation for the scalar dissipation rate $\chi_{1}$, and a similar trend is found to the above non-reacting strong puffing case.

\section{Acknowledgements}

The authors are grateful for the financial support of the Engineering and Physical Sciences Research Council (EPSRC) of the UK under Grant No. EP/J018023/1.

\section{References}

[1] Dryer, F. L., 1977, Symposium (International) on Combustion, 16 (1), pp. 279-295.

[2] Law, C. K., 1982, Progress in Energy and Combustion Science, 8 (3), pp. 171-201.

[3] Kadota, T., Yamasaki, H., 2002, Progress in Energy and Combustion Science, 28 (5), pp. 385-404.

[4] Takewaki, H., Nishiguchi, A., Yabe, T., 1985, Journal of Computational Physics, 61 (2), pp. 261-268.

[5] Yabe, T., Xiao, F., Utsumi, T., 2001, Journal of Computational Physics, 169, pp. 556-593.

[6] Sussman, M., Smereka, P., Osher, S., 1994, Journal of Computational Physics, 114 (1), pp. 146-159.

[7] Kunigi, T., 1997, Transactions of the Japan Society of Mechanical Engineers Series B, 63, pp. 1576-1584.

[8] Brackbill, J. U., Kothe, D. B., Zemach, C., 1992, Journal of Computational Physics, 100 (2), pp. 335-354.

[9] Tanguy, S., Ménard, T., Berlemont, A., 2007, Journal of Computational Physics, 221 (2), pp. 837-853.

[10] Juric, D., Tryggvason, G., 1998, International Journal of Multiphase Flow, 24 (3), pp. 387-410.

[11] Shinjo, J., Xia, J., Ganippa, L. C., Megaritis, A., 2014, Physics of Fluids, 26, article no. 103302.

[12] Shinjo, J., Xia, J., Megaritis, A., Ganippa, L. C., Cracknell, R. F., 2016, Atomization and Sprays, 26 (6), pp. 551-583.

[13] Shinjo, J., Xia, J., Ganippa, L. C., Megaritis, A., 2016, Journal of Fluid Mechanics, 793, pp. 444-476.

[14] Shinjo, J., Xia, J., 2017, Proceedings of the Combustion Institute, 36 (2), pp. 2513-2521.

[15] Prosperetti, A., Plesset, M. S., 1984, Physics of Fluids, 27 (7), pp. 1590-1602.

[16] Sirignano, W. A., 1983, Progress in Energy and Combustion Science, 9 (4), pp. 291-322.

[17] Abramzon, B., Sirignano, W. A., 1989, International Journal of Heat and Mass Transfer, 32 (9), pp. 1605-1618.

[18] Bilger, R. W., 2011, Combustion and Flame, 158 (2), pp. 191-202.

[19] Hasse, C., Peters, N., 2005, Proceedings of the Combustion Institute, 30 (2), pp. 2755-2762. 